\title{
Cartilage Degeneration, Subchondral Mineral and Meniscal Mineral Densities in Hartley and Strain 13 Guinea Pigs
}

\author{
Yubo Sun ${ }^{*}, 1$, Brian P. Scannell ${ }^{1}$, Patrick R. Honeycutt ${ }^{1}$, David R. Mauerhan ${ }^{1}$, H. James Norton ${ }^{2}$ and \\ Edward N. Hanley Jr. ${ }^{1}$ \\ ${ }^{1}$ Department of Orthopedic Surgery, Cannon Research, Carolinas Medical Center, PO Box 32861, Charlotte, NC \\ 28232, USA \\ ${ }^{2}$ Dickson Advanced Analytics Group, Carolinas Medical Center, PO Box 32861, Charlotte, NC 28232, USA
}

\begin{abstract}
Osteoarthritis is a joint disease involved in articular cartilage, subchondral bone, meniscus and synovial membrane. This study sought to examine cartilage degeneration, subchondral bone mineral density (BMD) and meniscal mineral density (MD) in male Hartley, female Hartley and female strain 13 guinea pigs to determine the association of cartilage degeneration with subchondral BMD and meniscal MD. Cartilage degeneration, subchondral BMD and meniscal MD in 12 months old guinea pigs were examined with histochemistry, X-ray densitometry and calcium analysis. We found that male Hartley guinea pigs had more severe cartilage degeneration, subchondral BMD and meniscal MD than female Hartley guinea pigs, but not female strain 13 guinea pigs. Female strain 13 guinea pigs had more severe cartilage degeneration and higher subchondral $\mathrm{BMD}$, but not meniscal $\mathrm{MD}$, than female Hartley guinea pigs. These findings indicate that higher subchondral BMD, not meniscal $\mathrm{MD}$, is associated with more severe cartilage degeneration in the guinea pigs and suggest that abnormal subchondral BMD may be a therapeutic target for OA treatment. These findings also indicate that the pathogenesis of OA in the male guinea pigs and female guinea pigs are different. Female strain 13 guinea pig may be used to study female gender-specific pathogenesis of OA.
\end{abstract}

Keywords: Guinea pigs, meniscus, mineral density, osteoarthritis, subchondral bone.

\section{INTRODUCTION}

Osteoarthritis (OA) is a degenerative disease of articular joints characterized by focal areas of cartilage degeneration, meniscal abnormalities and bony changes. It is now well accepted that $\mathrm{OA}$ is not merely an articular cartilage disease, but a disease of the whole joint. An important local factor that may play a role in $\mathrm{OA}$ is the biochemical or biomechanical property of subchondral bone. Clinical examinations found that subchondral bone in OA patients were abnormal $[1,2]$. Subchondral bone mineral density (BMD) was substantially higher in OA patients compared to control subjects and high subchondral BMD was associated with cartilage defects, joint space narrowing, subchondral sclerosis, osteophytes and meniscal damages [3-7]. In addition, laboratory studies found that explants and primary cultured osteoblasts derived from bone tissues of $\mathrm{OA}$ patients secreted high level of urokinase plasminogen activator, a serine protease capable of degrading the cartilage $[8,9]$. All these findings indicate that abnormal biochemical and biomechanical alterations in the subchondral bone of OA patients may play an important role in the development and/or progression of OA.

*Address correspondence to this author at the Department of Orthopedic Surgery, Cannon Research 303, Carolinas Medical Center, PO Box 32861, Charlotte, NC 28232, USA; Tel: 704/355-5656; Fax: 704/355-5620;

E-mail: yubo.sun@carolinashealthcare.org
Another important local factor that may play a role in OA is the structural integrity or biochemical property of knee menisci. Studies found that meniscal degeneration was a general feature of knee $\mathrm{OA}$ and contributed to joint space narrowing $[10,11]$. Meniscal lesions at baseline were more common in knees that developed OA than in knees that did not developed OA [12]. OA meniscal cells displayed a distinct gene expression profile different from normal meniscal cells [13]. The involvement of knee menisci in the development or progression of OA has also been highlighted by several findings that meniscal extrusion, vascular penetration and calcification were associated with severe cartilage lesions in patients with OA [14-17].

Because the whole joint is affected in OA, identification of the changes in the subchondral bone and knee menisci in addition to the articular cartilage may not only provide information valuable for a better understanding the pathogenesis of OA but also valuable for the development of tissue-specific disease modifying drugs for OA therapy. Hartley guinea pigs develop OA spontaneously. Articular cartilage in Hartley guinea pigs exhibits progressive degenerative changes that closely resemble human OA, including articular cartilage surface fibrillation, proteoglycan loss and osteophyte formation. Therefore, Hartley guinea pigs have been widely used to study the pathogenesis of OA and test disease modifying drugs. Previous studies found that male Hartley guinea pigs displayed severer cartilage degeneration than male strain 13 guinea pigs and that 
subchondral bone density and meniscal ossification in male Hartley guinea pigs were associated with cartilage degeneration, suggesting that abnormal subchondral bone metabolisms and abnormal meniscal ossification may play a role in the development or progression of OA in the Hartley guinea pigs $[18,19]$.

However, no studies have examined and compared the subchondral BMD and meniscal mineral density (MD) in the male Hartley guinea pigs and female Hartley guinea pigs although it is known that female Hartley guinea pigs have less severe cartilage degeneration than male Hartley guinea pigs [20]. In addition, no studies have examined and compared cartilage degeneration, subchondral BMD and meniscal MD in female Hartley guinea pigs and female strain 13 guinea pigs. In this study, we examined articular cartilage degeneration, subchondral BMD and meniscal MD in these guinea pigs to test the hypothesis that abnormal subchondral BMD or meniscal MD is associated with more severe cartilage degeneration. The information obtained from this study not only may be important for a better understanding of the pathogenesis of OA but also important for the development of tissue-specific disease modifying drugs for OA therapy.

\section{MATERIALS AND METHODOLOGY}

\section{Animals}

Male $(n=5)$ and female $(n=5)$ Hartley guinea pigs of 2 months old were obtained from Charles River Laboratories (Wilmington, MA). Female strain 13 guinea pigs $(n=10)$ of 2 months old were obtained from Army Research Laboratory (Adelphi, MD). Animals were individually housed in $29 \times 21$ x 10 inch solid bottom cages. Guinea pig chow (No. 5025; Ralston Purina, Richmond, Indiana) and water were available ad libitum. By 12 months of age these guinea pigs were anaesthetized with 5\% isoflurane (Isofluran, Baxter, Deerfield, IL) inhalation and euthanized by administration of $300 \mathrm{mg} / \mathrm{kg}$ Euthasol (Virbac Animal Health, Ft. Worth, Texas) intraperitoneally. Hind limbs were collected and knee joints were dissected for examinations. This study was conducted according to the guidelines set forth by the Institutional Animal Care \& Use Committee of Carolinas Medical Center, which reviewed and approved the protocol.

\section{Gross, Macroscopic and Histological Examinations}

Knee joints were dissected to separate femur, menisci and tibia. Tibia plateaus was first stained with India ink as described [21]. Digital images of India ink stained tibia plateaus were taken and observed macroscopically. The tibia plateaus were then fixed in neutral buffered $4 \%$ paraformaldehyde for two days and decalcified in Cal-Ex II solution (Fisher Scientific, Fairlawn, NJ) for five days. The decalcified tibia plateaus were cut sagittally to separate the medial and lateral tibia plateaus. The medial tibia plateau was cut again sagittally to remove portion of the plateau that was originally covered with meniscus. The resulting specimens were embedded in paraffin and sectioned with a Leica RM2025 microtome (Nussloch, Germany) to obtain 4 $\mu \mathrm{m}$ sections. Three non-consecutive sections per knee, obtained at $300 \mu \mathrm{m}$ intervals, were stained with safranin $\mathrm{O}$ and counter stained with fast green. These stained sections were photographed (4X magnification) using OPTIHHOT-2 microscope equipped with a digital camera (Nikon, Japan). Histologic scoring of these sections was performed according to Mankin criteria with modifications [18]. For each guinea pig, the final histologic score for the medial plateau cartilage was calculated as the mean of histologic score of 3 non-consecutive sections. Osteophytes, cellularity and the integrity of the tidemark were not included in the assessment because the incidence of osteophytes, cartilage cellularity and the integrity of tide mark were not observed to differ significantly between these guinea pigs.

\section{Subchondral BMD and Meniscal MD}

Measurements of subchondral BMD $\left(\mathrm{g} / \mathrm{cm}^{2}\right)$ were made with a Lunar PIXImus X-ray bone densitometer (GE Lunar, Madison, WI). The instrument was calibrated before each scanning session using a Phantom with known BMD according to the manufacturer's guidelines. Each tibia plateau was placed on the scanning surface at the same position and orientation, and scanned using software version 2.10.013 to determine subchondral BMD. An area of same size at same location of the subchondral bone $(2 \mathrm{~mm} \times 1$ $\mathrm{mm}$ ) in each medial tibia plateau immediately under the articular cartilage was assessed. Acquisition time per scan was approximately 5 minutes. Three repeated measurements were performed and the mean of 3 measurements was calculated. Similarly, each medial meniscus was placed on the scanning surface at the same position and orientation, and scanned to determine meniscal MD.

\section{Meniscal Calcium Content}

Meniscal calcium content was determined using atomic absorption spectrophometry following the procedure described [22]. Briefly, medial meniscus was placed in a tarred fused silica crucible, weighed, dried at $110^{\circ} \mathrm{C}$, and ashed for 24 hours at $650^{\circ} \mathrm{C}$. The ash was dissolved in $1 \mathrm{ml}$ concentrated $\mathrm{HCl}$, diluted, and assayed for calcium. Two blank crucibles were included in each assay.

\section{Statistical Analyses}

Data were presented as mean \pm standard deviation. The difference in histologic scores between 2 groups was analyzed using Wilcoxon rank-sum test. The difference in subchondral BMD, meniscal $\mathrm{MD}$ and meniscal calcium content between two groups was analyzed using Student's T test. In all cases, $\mathrm{p}<0.05$ was considered significant. Statistical analysis was performed using $\mathrm{SAS}^{\circledR}$ software, version 9.3 (SAS Institute Inc., Cary, NC).

\section{RESULTS}

\section{Gross, Macroscopic and Histological Examinations}

Representative images of Indian ink-stained tibia plateaus were shown in Fig. (1). It was clear that severe articular cartilage degeneration occurred in the medial tibia plateaus but not in the lateral tibia plateaus, and that cartilage degeneration was mainly occurred in the central part of the medial tibia plateaus (arrows). Male Hartley guinea pigs and 


\section{Male $\mathrm{H}$}

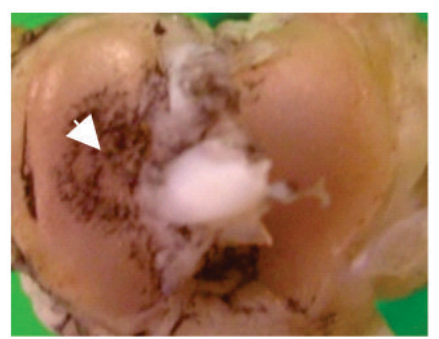

Female $\mathrm{H}$

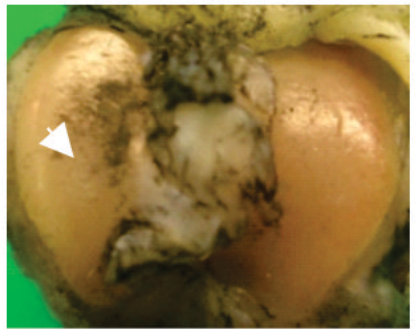

Female S-13

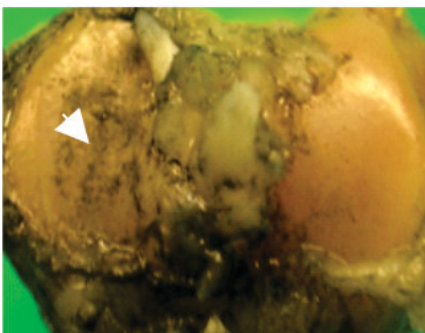

Fig. (1). Representative images of Indian ink stained tibia plateaus (magnification 5X). Indian ink stained tibia plateau of a male Hartley guinea pig (Male H), a female Hartley guinea pig (Female $\mathrm{H}$ ) and a female strain 13 guinea pig (Female S-13). Arrows - central part of medial tibia plateau that was not covered with meniscus.

female strain 13 guinea pigs appeared to having more severe cartilage degeneration than the age-matched female Hartley guinea pigs.

Representative safranin O-stained sections of medial tibia plateaus of 3 male Hartley guinea pigs, female Hartley guinea pigs and 3 female strain 13 guinea pigs were provided in Fig. (2). Consistent with the gross appearances of the medial tibia plateaus shown in Fig. (1), male Hartley guinea pigs and female strain 13 guinea pigs displayed more severe articular surface fibrillation and cartilage clefts than the aged-matched female Hartley guinea pigs. In the male Hartley guinea pigs, cartilage clefts extended into the middle and deep zones of articular cartilage while in female Hartley guinea pigs, cartilage clefts only occurred in the superficial zone. Male Hartley guinea pigs also displayed more severe proteoglycan loss (loss of safranin-O staining) than the agedmatched female Hartley guinea pigs. In the male Hartley guinea-pigs, proteoglycan loss occurred across all the 4 zones (superficial, middle, deep and calcified zones) whereas in female Hartley guinea pigs, proteoglycan loss occurred mainly in the superficial zone, and in some cases, in the middle zone. Similar to the male Hartley guinea pigs, cartilage clefts extended into middle and deep zones, and proteoglycan loss occurred in all 4 zones in the female strain 13 guinea pigs.

The mean histologic scores of medial tibia plateau cartilage in male Hartley guinea pigs, female Hartley guinea pigs and female strain 13 guinea pigs were determined and presented in Fig. (3). As shown, both the histologic scores of the medial tibia plateau cartilage in the male Hartley guinea
Male H
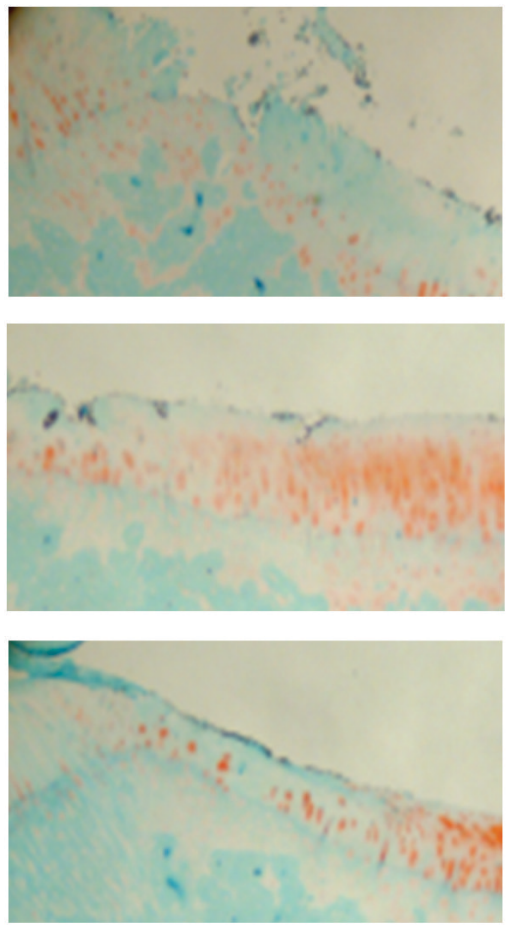

Female H
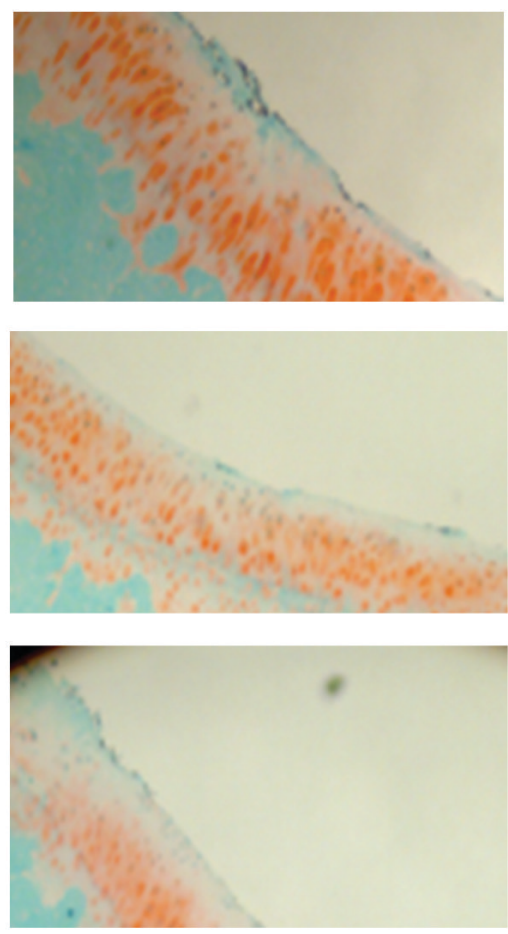

Female S-13
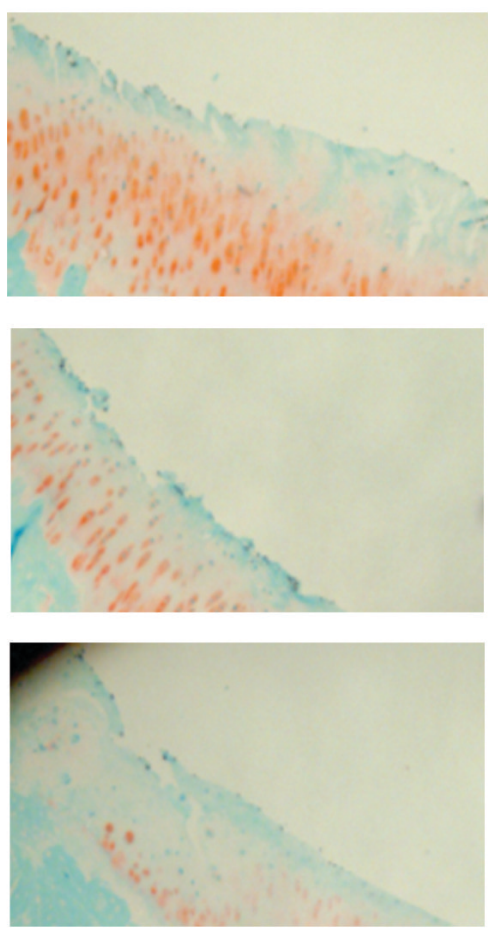

Fig. (2). Safranin O-stained sections of medial tibia plateaus (magnification 20X). Left photos: sections of medial tibia plateaus of 3 male Hartley guinea pigs. Middle photos: sections of medial tibia plateaus of 3 female Hartley guinea pigs. Right photos: sections of medial tibia plateaus of 3 female strain 13 guinea pigs. 
pigs $(=8.70)$ and female strain 13 guinea pigs $(=7.05)$ were higher than the histologic score of the medial tibia plateau cartilage in the female Hartley guinea pigs $(=4.70)$. The differences in the histologic scores between the male Hartley guinea pigs and female Hartley guinea pigs was statistically significant $(\mathrm{p}=0.016)$. The differences in the histologic scores between the female strain 13 guinea pigs and female Hartley guinea pigs was also statistically significant $(\mathrm{p}=$ 0.018). Although the histological score of the medial tibia plateau cartilage in the male Hartley guinea pigs was higher than that in female strain 13 guinea pigs, the difference did not reach to significance $(p=0.379)$.

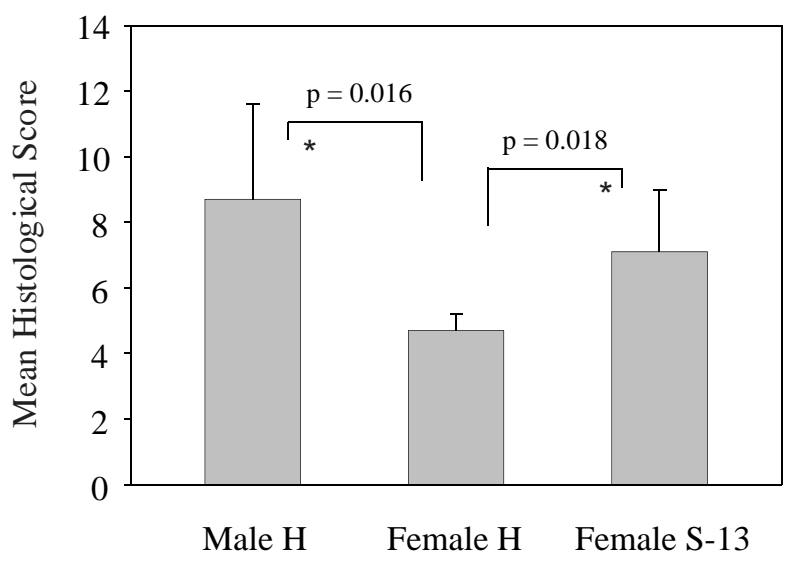

Fig. (3). Mean histological scores of medial plateau cartilage. Left bar: mean histologic score in male Hartley guinea pigs. Middle bar: mean histologic score in female Hartley guinea pigs. Right bar: mean histologic score in female strain 13 guinea-pigs. ${ }^{*}=\mathrm{P}<0.05$ versus female Hartley guinea pigs.

\section{Subchondral BMD}

Subchondral BMD in male Hartley, female Hartley and female strain 13 guinea-pigs were determined and the results were presented in Fig. (4). As shown, the subchondral BMD in male Hartley guinea pigs $\left(=0.3223 \mathrm{~g} / \mathrm{cm}^{2}\right)$ and female strain 13 guinea pigs $\left(=0.3113 \mathrm{~g} / \mathrm{cm}^{2}\right)$ were similar, and both were higher than the subchondral BMD in female Hartley guinea pigs $\left(=0.2881 \mathrm{~g} / \mathrm{cm}^{2}\right)$. The difference in the subchondral BMD between male Hartley and female Hartley guinea pigs was significant $(\mathrm{P}=0.036)$. The difference in the subchondral BMD between female strain 13 and female Hartley guinea pigs was also statistically significant $(\mathrm{p}=$ 0.049). Although the subchondral BMD in the male Hartley guinea pigs was higher than that in the female strain 13 guinea pigs, the difference did not reach to significance $(\mathrm{p}=$ $0.221)$.

\section{Meniscal MD}

Meniscal MD in male Hartley, female Hartley and female strain 13 guinea-pigs were determined and the results were presented in Fig. (5). As shown, the mean meniscal MD in the female Hartley guinea pigs $\left(=0.353 \mathrm{~g} / \mathrm{cm}^{2}\right)$ and female strain 13 guinea pigs $\left(=0.345 \mathrm{~g} / \mathrm{cm}^{2}\right)$ were similar, and both were lower than the mean meniscal MD in the male Hartley guinea pigs $\left(=0.442 \mathrm{~g} / \mathrm{cm}^{2}\right)$. The difference in the meniscal MD between female Hartley and male Hartley guinea pigs was statistically significant $(\mathrm{p}=0.019)$. The difference in the meniscal MD between female strain 13 and male Hartley guinea pigs was also statistically significant $(\mathrm{p}=0.001)$.

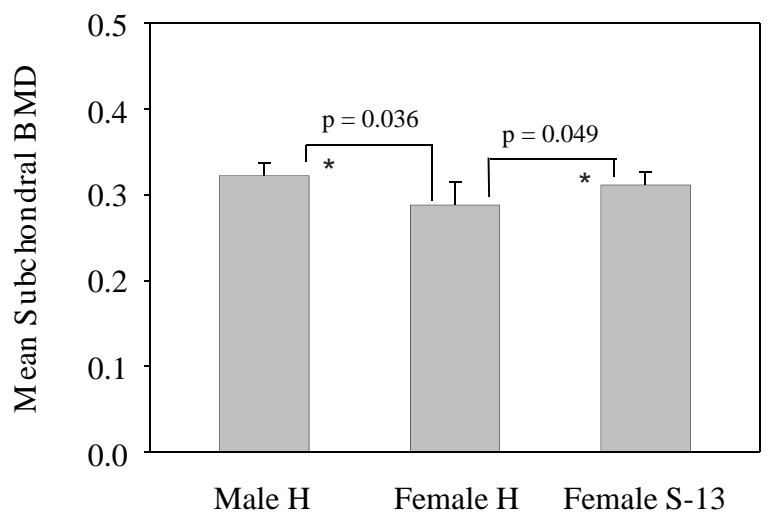

Fig. (4). Mean subchondral BMD. Left bar: mean subchondral BMD in male Hartley guinea pigs. Middle bar: mean subchondral BMD in female Hartley guinea pigs. Right bar: mean subchondral $\mathrm{BMD}$ in female strain 13 guinea pigs. ${ }^{*}=\mathrm{P}<0.05$ versus female Hartley guinea-pigs.

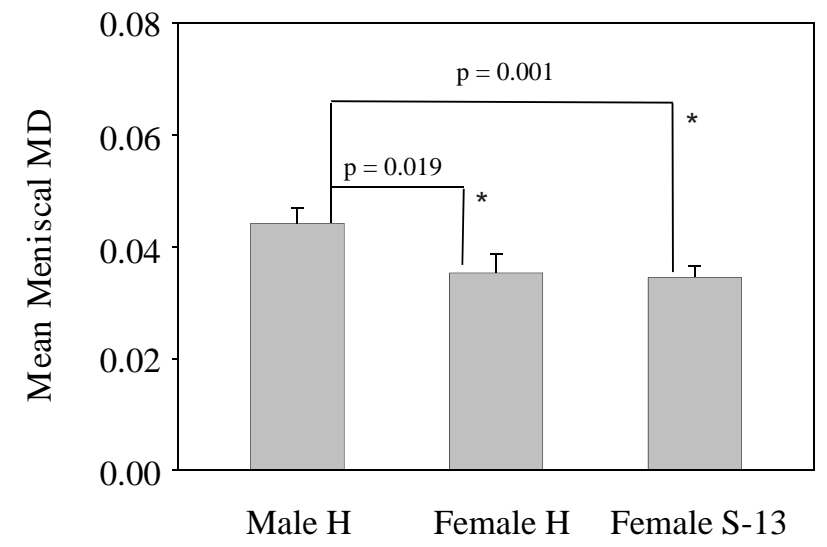

Fig. (5). Mean meniscal MD. Left bar: mean meniscal MD in male Hartley guinea pigs. Middle bar: mean meniscal MD in female Hartley guinea pigs. Right bar: mean meniscal MD in female strain 13 guinea pigs. ${ }^{*}=\mathrm{P}<0.05$ versus male Hartley guinea pigs.

\section{Meniscal Calcium Content}

We performed chemical analysis to determine and compare the calcium content in the medial meniscus of all these guinea pigs and the results are presented in Fig. (6). Consistent with the results obtained with X-ray densitometry, the meniscal calcium content in female Hartley guinea pigs (= $1.3172 \mathrm{mg} /$ meniscus $)$ and female strain 13 guinea pigs (= $1.3347 \mathrm{mg} /$ meniscus) were similar, and both were lower than the meniscal calcium content in the male Hartley guinea pigs (= $1.511 \mathrm{mg} / \mathrm{meniscus})$ although these differences did not reach statistical significance due to large variations in each individual calcium measurement. 


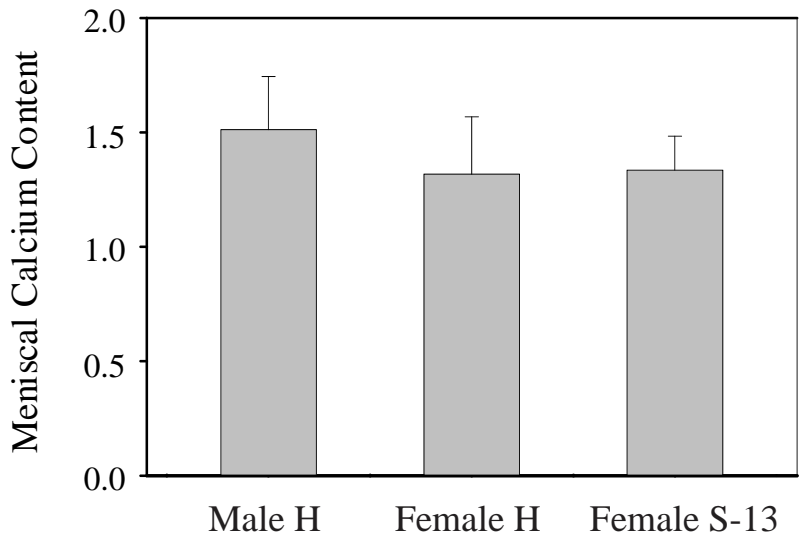

Fig. (6). Mean meniscal calcium content. Left bar: meniscal calcium content in male Hartley guinea pigs. Middle bar: mean meniscal calcium content in female Hartley guinea pigs. Right bar: mean meniscal calcium content in female strain 13 guinea pigs.

\section{DISCUSSION}

We demonstrate that not only the male Hartley guinea pigs, female strain 13 guinea pigs also display more severe cartilage degeneration than female Hartley guinea pigs. It was believed that the less severe cartilage degeneration in female Hartley guinea pigs compared to male Hartley guinea pigs was due to body weight difference [20]. Male Hartley guinea pigs by 12 months of age have a body weight between 1000 grams and 1300 grams whereas female Hartley guinea pigs by 12 months of age have a body weight between 900 grams and 1150 grams. However, the fact that female strain 13 guinea pigs, which have a smaller body weight (between 700 grams and 920 grams) (Fig. 7), display more severe cartilage degeneration than the age-matched female Hartley guinea pigs indicates that body weight is not a key determining factor to the development and/or progression of OA in these guinea pigs. Our finding doesn't exclude that body weight may play a role in OA severity.

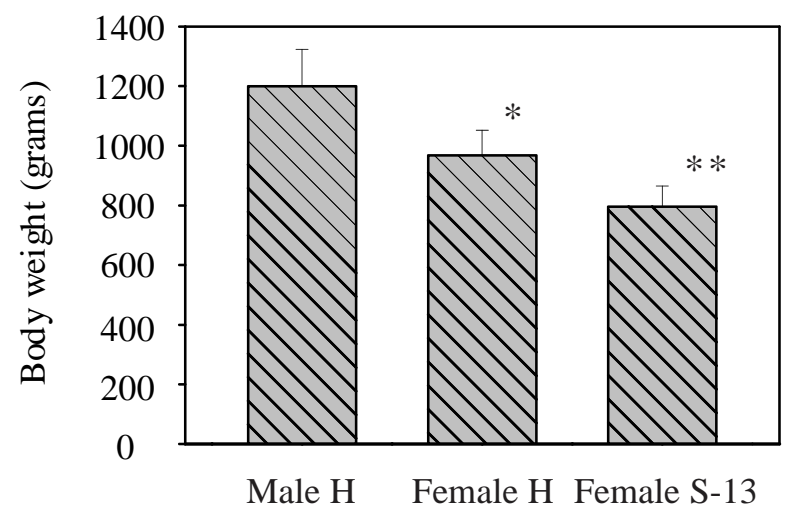

Fig. (7). Mean body weight. Left bar: body weight in male Hartley guinea pigs. Middle bar: body weight in female Hartley guinea pigs. Right bar: body weight in female strain 13 guinea pigs. $*=\mathrm{P}$ $<0.05$ versus male Hartley guinea pigs. ${ }^{*} *=\mathrm{P}<0.05$ versus both male and female Hartley guinea pigs.

We found that the histologic score of cartilage degeneration and subchondral BMD in male Hartley guinea pigs were significantly higher than those in female Hartley guinea pigs. To the best of our knowledge, this is the first study to report that male Hartley guinea pigs have higher subchondral BMD than the female Hartley guinea pigs, which displayed less severe OA compared to male Hartley guinea pigs. We also found both the histologic score of cartilage degeneration and subchondral BMD in female strain 13 guinea pigs were significantly higher than those in female Hartley guinea pigs. This positive association between cartilage degeneration and subchondral BMD provides support for the notion that subchondral bone may play a role in the development and/or progression of OA and is potentially a key target for OA therapy [23]. The changes in subchondral BMD may affect the biomechanical properties of the subchondral bone and result in a loss of subchondral bone shock adsorbing capacity [24]. The changes in subchondral BMD may also affect the cytokines and growth factors produced in the subchondral bone, which may seep through the subchondral bone-cartilage interface and promote cartilage breakdown [25].

Most previous studies suggest that high subchondral BMD may play a pathological role in the development and/or progression of OA, however, a couple of studies found that high subchondral BMD decreased the risk of progression of radiographic $\mathrm{OA}$ and protected cartilage against degeneration [26, 27]. These contradicting findings indicate $\mathrm{OA}$ is a heterogeneous disease. A subset of $\mathrm{OA}$ patients with high subchondral BMD may benefit from treatment with bone acting anti-mineralization agents and a subset of OA patients may benefit from treatment with boneacting anti-resorptive agents. The guinea pig model of OA is a useful model for testing the effects of small molecules that inhibit (or promote) subchondral bone mineralization on the development and/or progression of OA.

Meniscal ossification increases with age and correlates with articular damages in male Hartley guinea pigs [19]. In this study, we found that both the histologic score of cartilage degeneration and meniscal MD in male Hartley guinea pigs were higher than those in female Hartley guinea pigs. These findings suggest that abnormal meniscal ossification may play a role in $\mathrm{OA}$ in the Hartley guinea pigs. However, the finding that female strain 13 and female Hartley guinea pigs had similar meniscal MD contradicts with the notion that abnormal meniscal ossification plays a role in OA. It is worth noting that Walton found that two closely related strains of mice, STR/ORT and CBA/ORT, both displayed meniscal ossification, but only STR/ORT mice developed OA [28]. The finding presented in this study together with the previous findings [28] indicate that the role of meniscal ossification in animal OA is more complex, and may be strain-specific or gender-dependent. Nevertheless, meniscal ossification does not appear to be a parameter of paramount importance in the pathogenesis of $\mathrm{OA}$ in these guinea pigs. Guinea pig model of OA may be not a good model for the study of the role of meniscal ossification in OA $[17,29]$.

\section{CONCLUSION}

Higher subchondral BMD, but not meniscal MD, is associated with more severe cartilage degeneration in guinea pigs, suggesting that abnormal subchondral bone 
metabolisms may play a role in the development and/or progression of OA. The pathogenesis of OA in guinea pigs is gender-specific. Female strain 13 guinea pigs, together with female Hartley guinea pigs, may be used to study the female gender-specific pathogenesis of $\mathrm{OA}$ and/or the role of sex hormone in the development of OA.

\section{ABBREVIATIONS}

$$
\begin{array}{ll}
\mathrm{BMD} & =\text { Bone mineral density } \\
\mathrm{MD} & =\text { Mineral density } \\
\mathrm{OA} & =\text { Osteoarthritis }
\end{array}
$$

\section{CONFLICT OF INTEREST}

The authors confirm that this article content has no conflict of interest.

\section{ACKNOWLEDGEMENTS}

This study is supported in part by a CharlotteMecklenburg Health Science Foundation Grant and a Mecklenburg County Medical Society Smith Arthritis Fund Grant (to YS). This study was performed at Carolinas Medical Center, Charlotte, NC. The authors would like to thank Natalia Zinchenko for her help with histological examinations and calcium content analysis.

\section{REFERENCES}

[1] Herrero-Beaumont G, Roman-Blas JA, Largo R, et al. Bone mineral density and joint cartilage: four clinical settings of a complex relationship in osteoarthritis. Ann Rheum Dis 2011; 70: 1523-5.

[2] Funck-Brentano T, Cohen-Solal M. Subchondral bone and osteoarthritis. Curr Opin Rheumatol 2015; 27: 420-6.

[3] Bruyere O, Dardenne C, Lejeune E, et al. Subchondral tibial bone mineral density predicts future joint space narrowing at the medial femoro-tibial compartment in patients with knee osteoarthritis. Bone 2003; 32: 541-5.

[4] Clarke S, Wakeley C, Duddy J, et al. Dual-energy X-ray absorptiometry applied to the assessment of tibial subchondral bone mineral density in osteoarthritis of the knee. Skeletal Radiol 2004; 33: 588-95.

[5] Dore D, Ding C, Jones G. A pilot study of the reproducibility and validity of measuring knee subchondral bone density in the tibia. Osteoarthr Cartil 2008; 16: 1539-44.

[6] Hardcastle SA, Dieppe P, Gregson CL, et al. Individuals with high bone mass have an increased prevalence of radiographic knee osteoarthritis. Bone 2015; 71: 171-9.

[7] Lo GH, Niu J, McLennan CE, et al. Meniscal damage associated with increased local subchondral bone mineral density: a Framingham study. Osteoarthr Cartil 2008; 16: 261-7.

[8] Hilal G, Martel-Pelletier J, Pelletier JP, et al. Osteoblast-like cells from human subchondral osteoarthritic bone demonstrate an altered phenotype in vitro: possible role in subchondral bone sclerosis. Arthritis Rheum 1998; 41: 891-9.

[9] Lajeunesse D. Altered subchondral osteoblast cellular metabolism in osteoarthritis: cytokines, eicosanoids, and growth factors. J Musculoskelet Neuronal Interact 2002; 2: 504-6.
[10] Bennett LD, Buckland-Wright JC. Meniscal and articular cartilage changes in knee osteoarthritis: a cross-sectional double-contrast macroradiographic study. Rheumatology 2002; 41: 917-23.

[11] Hunter DJ, Zhang YQ, Tu X, et al. Change in joint space width: hyaline articular cartilage loss or alteration in meniscus? Arthritis Rheum 2006; 54: 2488-95.

[12] Englund M, Guermazi A, Roemer FW, et al. Meniscal tear in knees without surgery and the development of radiographic osteoarthritis among middle-aged and elderly persons: The Multicenter Osteoarthritis Study. Arthritis Rheum 2009; 60: 831-9.

[13] Sun Y, Mauerhan D, Hanley EN, Gruber H. Analysis of meniscal degeneration and meniscal gene expression. BMC Musculoskelet Disord 2010; 11:19.

[14] Lee DH, Lee BS, Kim JM, et al. Predictors of degenerative medial meniscus extrusion: radial component and knee osteoarthritis. Knee Surg Sports Traumatol Arthrosc 2011; 19: 222-9.

[15] Wang Y, Wluka AE, Pelletier JP, et al. Meniscal extrusion predicts increases in subchondral bone marrow lesions and bone cysts and expansion of subchondral bone in osteoarthritic knees. Rheumatology (Oxford) 2010; 49: 997-1004.

[16] Ashraf S, Wibberley H, Mapp PI, et al. Increased vascular penetration and nerve growth in the meniscus: a potential source of pain in osteoarthritis. Ann Rheum Dis 2011; 70: 523-9.

[17] Sun Y, Mauerhan DR, Honeycutt PR, et al. Calcium deposition in osteoarthritic meniscus and meniscal cell culture. Arthritis Res Ther 2010; 12: R56.

[18] Huebner JL, Hanes MA, Beekman B, et al. A comparative analysis of bone and cartilage metabolism in two strains of guinea-pig with varying degrees of naturally occurring osteoarthritis. Osteoarthr Cartil 2002; 10: 758-67.

[19] Thomsen J, Straarup T, Danielsen C, et al. Relationship between articular cartilage damage and subchondral bone properties and meniscal ossification in the Dunkin Hartley guinea pig model of osteoarthritis. Scand J Rheumatol 2011; 40: 391-9.

[20] Bendele AM. Animal models of osteoarthritis. J Musculoskelet Neuronal Interact 2001, 1: 363-76.

[21] Meachim G. Light microscopy of Indian ink preparations of fibrillated cartilage. Ann Rheum Dis 1972; 31: 457-64.

[22] Kay MA, Meyer MH, Delzer PR, Meyer RA, Jr. Changing patterns of femoral and skeletal mineralization during growth in juvenile $\mathrm{X}$ linked hypophosphatemic mice. Miner Electrolyte Metab 1985; 11: 374-80.

[23] Castaneda S, Roman-Blas JA, Largo R, Herrero-Beaumont G Subchondral bone as a key target for osteoarthritis treatment. Biochem Pharmacol 2012; 83: 315-23.

[24] Radin EL, Rose RM. Role of subchondral bone in the initiation and progression of cartilage damage. Clin Orthop Relat Res 1986; 3440.

[25] Westacott CI, Webb GR, Warnock MG, et al. Alteration of cartilage metabolism by cells from osteoarthritic bone. Arthritis Rheum 1997; 40: 1282-91.

[26] Zhang Y, Hannan MT, Chaisson CE, et al. Bone mineral density and risk of incident and progressive radiographic knee osteoarthritis in women: the Framingham Study. J Rheumatol 2000; 27: 1032-7.

[27] Cao Y, Stannus OP, Aitken D, et al. Cross-sectional and longitudinal associations between systemic, subchondral bone mineral density and knee cartilage thickness in older adults with or without radiographic osteoarthritis. Ann Rheum Dis 2014; 73: 2003-9.

[28] Walton M. Degenerative joint disease in the mouse knee; histological observations. J Pathol 1977; 123: 109-22.

[29] Sun Y, Mauerhan DR. Meniscal calcification, pathogenesis and implications. Curr Opin Rheumatol 2012; 24: 152-7. 